\title{
Synthesis of Some Substituted Furans and Endoperoxides Via Singlet Oxygen Reactions
}

\author{
Noor M. Mahdi ${ }^{1}$ and Yassir S. M. Al-Jawaheri ${ }^{*}$ \\ ${ }^{1,2^{*}}$ Department of Chemistry, College of Education for Pure Sciences, University of Mosul, Mosul, Iraq \\ E mail: ${ }^{1}$ nn0071667@gmail.com 2* ${ }^{*}$ yassir_chem71@uomosul.edu.iq
}

(Received August 02, 2021; Accepted October 26, 2021; Available online December 01, 2021)

DOI: 10.33899/edusj.2021.131010.1183, () 2021, College of Education for Pure Science, University of Mosul.

This is an open access article under the CC BY 4.0 license (http://creativecommons.org/licenses/by/4.0/)

\begin{abstract}
Cinnamaldehyde was used to condense with substituted acetophenone then with acetone to form substituted 2,4- diene -1- pentanone (1-4), after two moles of cinnamaldehyde condense with one mole of acetone to form 1,5- diphenyl nona- 8- tetraene -5 - one (5), for these reactions, sodium hydroxide was used as a catalyst, the resulted compounds reduced by Luche (specific reduction agent use cerium chloride as a catalyst with sodium borohydride to protect double bond) reaction to form 2,4- diene-1methoxy pentane (6-9) and -5- methoxy nona- 1,3,6,8 - tetraene - 1,9 - diyl dibenzene (10), the resulted dienes compound converted to endoperoxides (11-15) via singlet oxygen reaction in the present of Rose Bengal as a catalyst with light in chlorinated solvent and finally by appling of Appel reaction condition these compounds converted to furans (16-20). All mechanisms of the reaction were listed. These compounds were identified by thin layer chromatography TLC, and by their physical properties in addition, the IR spectroscopy and ${ }^{1}$ HNMR.
\end{abstract}

$\underline{\text { Keywords: }}$ Rose Bengal catalyst, singlet oxygen, endoperoxide, furan.

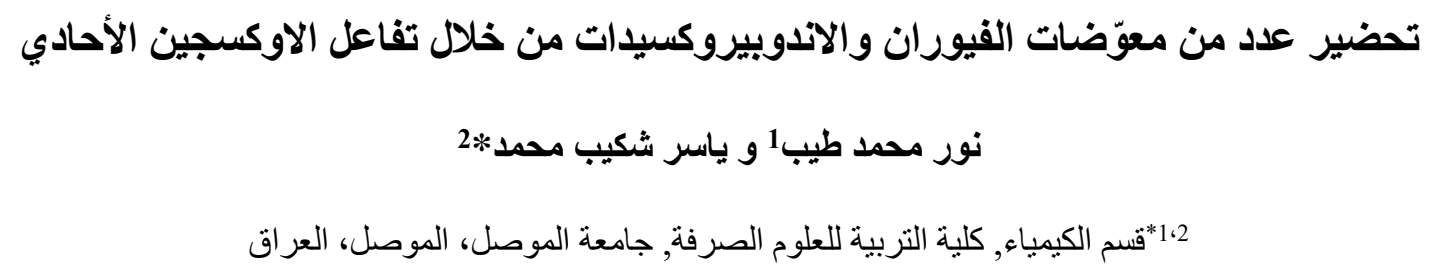

الخلاصة

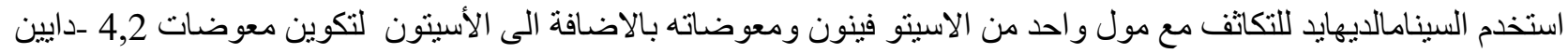

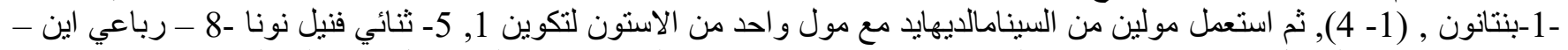

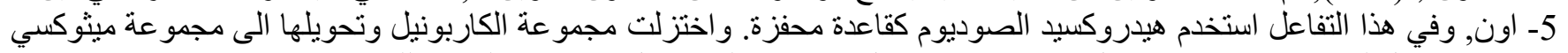

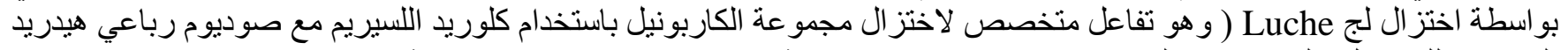

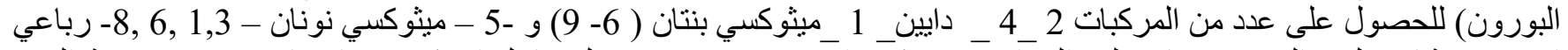

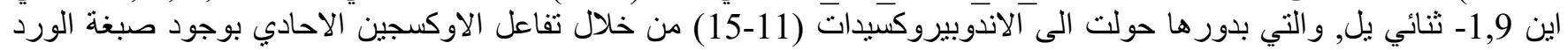
البنغالي مع الضوء في مذيبات مكلوره واخيرا حولت هذه المركبات الى الفيور انات المقابلة (16- 20) من خلال تطبيق ظروف الارك تفاعل 
ابيلAppel. ميكانيكيات التفاعل ادرجت في البحث. شخصت المركبات المحضرة بالطرق الفيزيائية وبأستخدام كروموتوكر افيا الطبقة

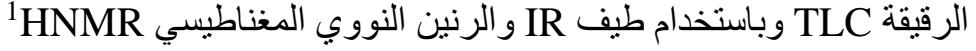

الكلمات الافتتاحية: صبغة الورد البنغالي, الاوكسجين الاحادي, الاندوبيروكسايد, الفيوران.

المقدمة Introduction

الفيوران يعتبر جزءاً مهماً في العديد من المكونات الصيدلانية والمركبات النشطة دوائيًا ومشتقاته التي تم الحصول عليها من الفوران المصنع مختبريا والموجودة في الطبيعية والتي تعتبر شائعة الاستخدام ولها مجموعة واسعة من المواد الصيدلانية بسبب الأنشطة البيولوجية الشكل (1) يوضح بعض الادوية المستعملة لعلاج بعض الامراض [1]. أظهرت مشتقات الفوران نشاطًا بيولوجيًا مثيرًا للاهتمام مثل مبيد الحشرات [2] ، مضاد للجراثيم [2] · مضاد للفطريات ,مضاد للفيروسات ، مضاد للأكسدةوضاد للألتهاب وكعقار

مسكن للألام [3].<smiles>NC(=O)N/N=C/c1ccc([N+](=O)[O-])o1</smiles><smiles>CCOCCOC(=O)c1ccc(/C=N/N2CCOC2=O)o1</smiles><smiles>O=C1CN(/N=C/c2ccc([N+](=O)[O-])o2)C(=O)N1</smiles>

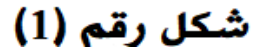

يمكن تحضير معوضات الفيوران بطرق عدة مثل مفاعلة مركبات 4,1- ثنائي الكيتون بوجود خامس اوكسيد الفسفور وحامض الكبريتيك وكذلك عن طريق مفاعلة ايثايل اسيتو استيت بوجود اليود وغيرها [4]. احد احدث طرق تحضير الفيوران ماتم نشره عام

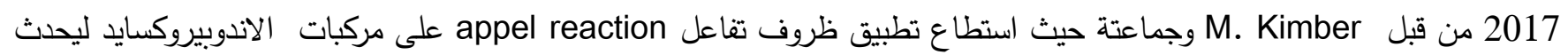

سحب جزية ماء مكونا الفيورانات المقابلة ليحضر عدد من مركبات فيورانات الاحماض الدهنية [5].<smiles>[R]C=CC=C[R]</smiles>

\section{1,3-diene}<smiles>[R]C1C=CC([R])OO1</smiles>

endoperoxide

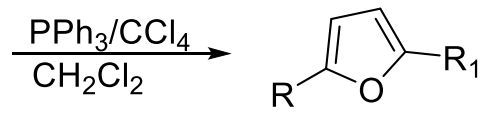

furan

الاندوبيركسايد يعرف ايضا ب dioxapropellanes وهي مركبات سداسية الحلقة وانها واحدة من اهم نواتج الاضافة [4+2] للأوكسجين الاحادي على الاواصر المتعاقبة للدأيين , هذه المركبات تمتلك خواص كيميائية وبيولوجية فريدة ومن اهمها مركب الارتيميزيا Artemisinin والذي يعتبر العلاج الفعال للقضاء على الملاريا [6], وكذلك وجود هذه المركبات في النباتات مثل المشرومMushroom الذي استخلص منه مجموعة من المركبات الفعاله [7]. يستخدم تفاعل الاوكسجين الاحادي

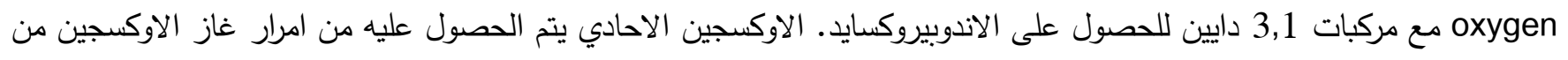

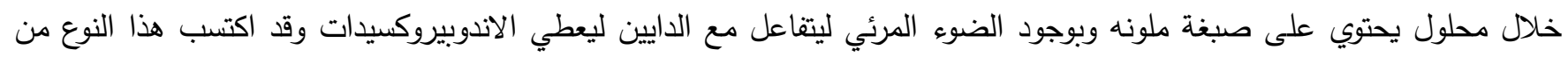
التفاعلات اهميه كبيرة في الآونة الأخيرة لاهميته في الحصول على مركبات بمعوضات وبودي مختلفة لها تطبيقات حياتية واسعة [8]. 
Fourier Transform Infrared spectrophotometer model shimadzu-FTIR-8400S Bruker Alpha FTIR, Germany, Tensor_27 - جهاز قياس درجة الانصهار (m.p. Electrothermal Melting Point Apparatus (Not Corrected)9300 ( جهاز ال)

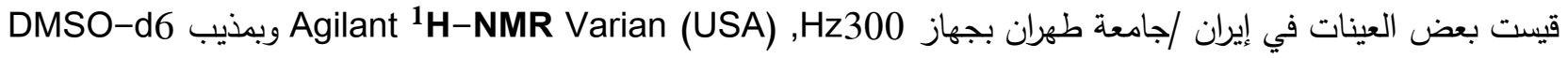

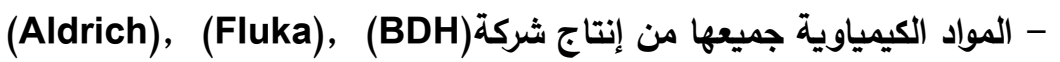
TLC كروموتوغرافيا الطبقة الرقيقة

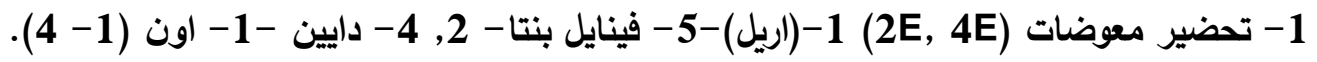
.[9-10] (2E,4E)-1-(aryl)-5-phenylpenta-2,4-diene-1-one

في دورق دائري اضيف (0.01mol.,1.23g ) من السينامالديهايد مع (.0.

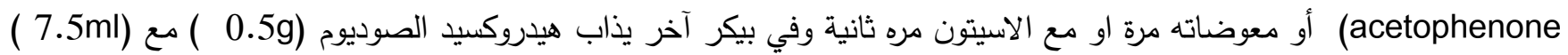
من الأيثانول ثم نضيفه الى محيط التفاعل الاول ويترك التفاعل على جهاز التحريك مدة (3) ساعات( يتم متابعة التفاعل

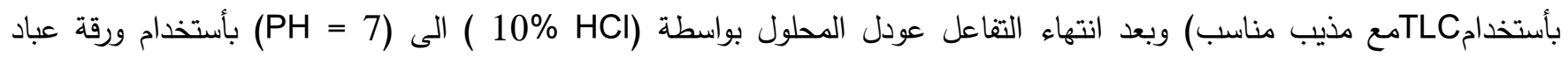
الشمس ثم يرشيح للحصول على راسب وتركه ليجف مدة (24) ساعة في درجة حرارة المختبر , تعاد بلورة الناتج باستخدام الأيثانول الساخن والماء والجدول رقم ( 1 ) يوضح الخواص الفيزيائية للنواتج.

\begin{tabular}{|c|c|c|c|c|c|c|c|}
\hline Comp.NO. & $\mathrm{Ar}$ & Molecular Formula & $\begin{array}{l}\text { M.Wt } \\
\text { g/mol. }\end{array}$ & $\begin{array}{c}\text { m.p. } \\
{ }^{\circ} \mathrm{C}\end{array}$ & $\begin{array}{c}\text { Yield } \\
\%\end{array}$ & Color & $\mathrm{R}_{\mathrm{f}}(10 \%$ EtOAc:\%90 Pet.ether) \\
\hline 1 & $p-\mathrm{CH}_{3}$ & $\mathrm{C}_{18} \mathrm{H}_{16} \mathrm{O}$ & 248 & 80_75 & $96 \%$ & أصفر & 0.3 \\
\hline 2 & $\mathrm{pH}$ & $\mathrm{C}_{17} \mathrm{H} 14 \mathrm{O}$ & 234 & $96-99$ & $80 \%$ & أصفر & 0.37 \\
\hline 3 & $p-\mathrm{Cl}$ & $\mathrm{C}_{17} \mathrm{H}{ }_{13} \mathrm{O} \mathrm{Cl}$ & 268 & 80_75 & $89 \%$ & 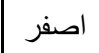 & 0.34 \\
\hline 4 & $\mathrm{CH}_{3}$ & $\mathrm{C}_{12} \mathrm{H}_{12} \mathrm{O}$ & 172 & 106 & $60 \%$ & بني & 0.3 \\
\hline
\end{tabular}

2- تحضير معوضات 1, 9- ثنائي فنيل نونان 1, 3, 6, 6, 8 -رباعي اين -5- اون/ (5). 1,9-diphenylnona-1,3,6,8-tetraen-5-one- 
استخدت الطريقة السابقة في تحضير المركب (5) وباستخدام مولين من السيناملديهايد مع مول واحد من الاسيتون ليعطي راسب اصفر درجة انصهاره 116 م وبنسبة 95\% وكانت Rf تساوي 0.32 في 10\% من الايثايل استيت في البتروليوم ايثر (40 الى مئى

3- تحضير معوضات - 5- ميثوكسي بنتان - 1, 3, - دايين - 1, 1, 5- ثنائي بنزين (6-110).

5-methoxy-5-substituted-penta1,3-dienyl]benzene

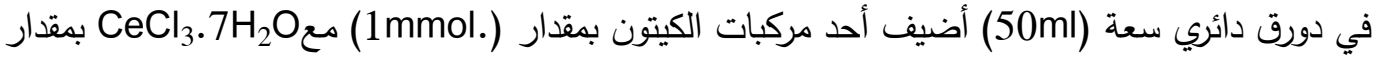

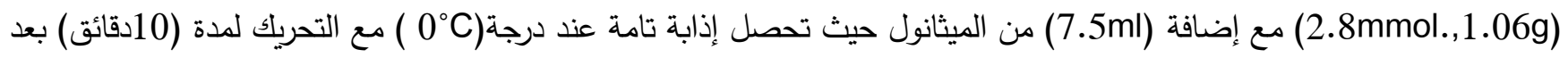
ذللك أضيفت تدريجياً صوديوم رباعي هيدريد البورون بمقدار (45mmol.1.71و) بشكل دفعات وبطيؤ لمدة ربع ساعة مع التحريك,

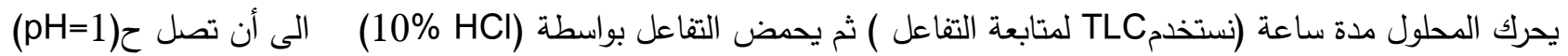

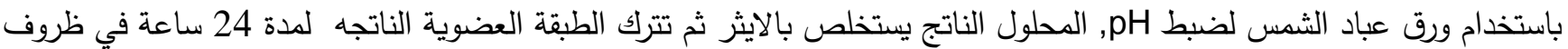
درجة حرارة المختبر (25 درجة مؤية) تعاد البلورة بواسطة الأيثانول الساخن والماء جدول رقم (2) يوضح الخواص الفيزياوية للمركبات الناتجة.

$$
\text { جدول (2) الخواص الفيزياوية للمركبات (6- 10) }
$$

\begin{tabular}{|c|c|c|c|c|c|c|c|}
\hline $\begin{array}{l}\text { Com } \\
\text { p.NO }\end{array}$ & $R$ & \begin{tabular}{|l|} 
Molecular \\
Formula
\end{tabular} & $\begin{array}{l}\text { M.Wt } \\
\text { g/mol. }\end{array}$ & $\begin{array}{l}\text { M.p. } \\
\left({ }^{\circ} \mathrm{C}\right)\end{array}$ & Color & \begin{tabular}{|l|l} 
Yield \\
$\%$ \\
\end{tabular} & $\begin{array}{l}\mathbf{R}_{\mathbf{f}} \\
/ \mathbf{1 0} \% \text { ether: } \\
90 \% \text { hexane }\end{array}$ \\
\hline 6 & $p-\mathrm{PhCH}_{3}$ & $\mathrm{C}_{19} \mathrm{H}_{20} \mathrm{O}$ & 264 & 145 & |أصفر فاتح | & 74 & 0.68 \\
\hline 7 & $\mathrm{Ph}$ & $\mathrm{C}_{18} \mathrm{H}_{18} \mathrm{O}$ & 250 & 150 & أصفر & 66 & 0.68 \\
\hline 8 & $p$-PhCl & $\mathrm{C}_{18} \mathrm{H}_{17} \mathrm{ClO}$ & 284 & 68 & أصفرغامق & 56 & 0.63 \\
\hline 9 & $\mathrm{CH} 3$ & $\mathrm{C}_{13} \mathrm{H}_{16} \mathrm{O}$ & 188 & 109 & أصفر & 60 & 0.6 \\
\hline 10 & & $\mathrm{C}_{22} \mathrm{H}_{22} \mathrm{O}$ & 302 & 154 & أصفرفاتح جدا & 70 & 0.68 \\
\hline
\end{tabular}

4-تحضير معوضات ميثايل ( فينايل) (6- فينايل - 3, 6- كائي هايدرو -1, 2- دايوكسين -3 - يل ) اوكسونيوم (11-15) 3-[(4-substution )(methoxy) methy)]-6-phenyl-3,6-dihydro-1,2-dioxin .[12] في دورق دائري سعة (250 ml) تم وضع ( . (0.014mol) من الدايين الناتج بعد عملية الأختزال مع (35 mg) من

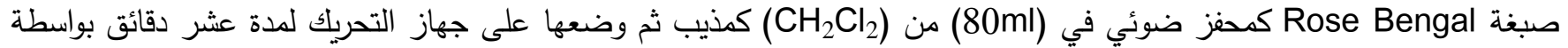

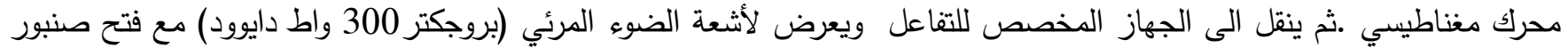

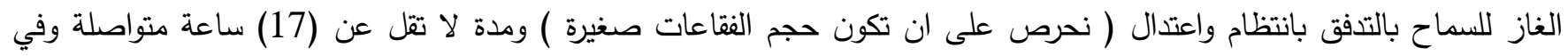
درجة حرارة الغرفة. يراقب سير التفاعل باستخدام كروماتوكرافيا الطبقة الرقيقة (TLC) بعد انتهاء التفاعل يبخر المذيب باستخدام الضغط 
المخلخل Rotary evaporator. تنقى المادة الناتجة في عمود الكروماتوكرافيا من خلال امرارها من طبقة 2سم من السيلكاجل (silica gel)

$$
\text { جدول رقم(3) يوضح الخواص الفيزياوية للمركبات (11- 15) }
$$

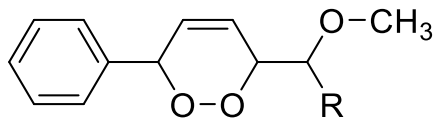

\begin{tabular}{|c|c|c|c|c|c|c|}
\hline Comp.NO. & $\mathbf{R}$ & Molecular Formula & $\begin{array}{l}\text { M.Wt } \\
\text { g/mol. }\end{array}$ & M.p & Color & $\begin{array}{l}R_{\mathrm{f}} / \\
10 \% \text { EtOAc: } \\
90 \% \text { Hexane }\end{array}$ \\
\hline 11 & $p-\mathrm{PhCH}_{3}$ & $\mathrm{C}_{19} \mathrm{H}_{20} \mathrm{O}_{3}$ & 296 & 120 & وردي فاتح & 0.4 \\
\hline 12 & $\mathrm{Ph}$ & $\mathrm{C}_{18} \mathrm{H}_{18} \mathrm{O}_{3}$ & 282 & 155 & احمر & 0.38 \\
\hline 13 & $p-\mathrm{PhCl}$ & $\mathrm{C}_{18} \mathrm{H}_{17} \mathrm{ClO}_{3}$ & 316 & 170 & 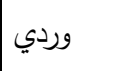 & 0.35 \\
\hline 14 & $\mathrm{CH}_{3}$ & $\mathrm{C}_{13} \mathrm{H}_{16} \mathrm{O}_{3}$ & 220 & 140 & وردي & 0.46 \\
\hline 15 & $\xi-1$ & $\mathrm{C}_{21} \mathrm{H}_{18} \mathrm{O}_{5}$ & 350 & 210 & وردي & 0.32 \\
\hline
\end{tabular}

5- تحضير معوضات الفيوران ( فينايل (5 - فينايل فيوران - 2- يل ) ميثوكسي ميثايل (16- 20) [5]. -2 (methoxy(sustituted)methyl)-5-phenylfuran

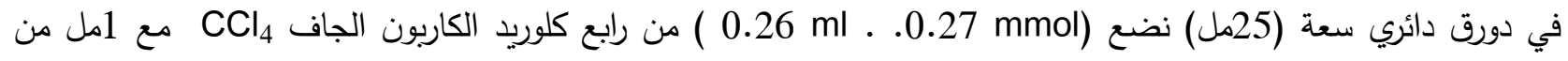
ثنائي كلوريد الكاربون الجاف لمدة ربع ساعة بواسطة المحرك المغناطيسي مع غلق فتحة الدورق. في دورق ثاني يذاب (mmol. 0.22) من الأندوبيروكسايد في

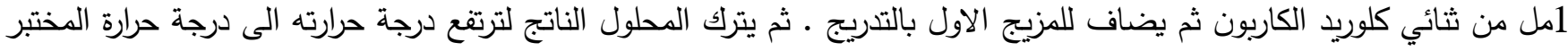

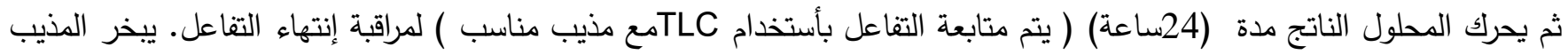
الناتج تحت الضغط المخلخل للحصول على مادة زيتية ذات لون أخضر فاتح, ينقى الناتج بواسطة عمود الكروماتوكرافيا بأمرار الناتج

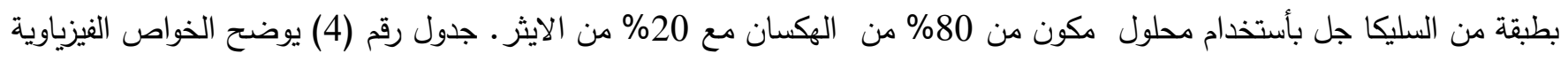
للمركبات الناتجة. 


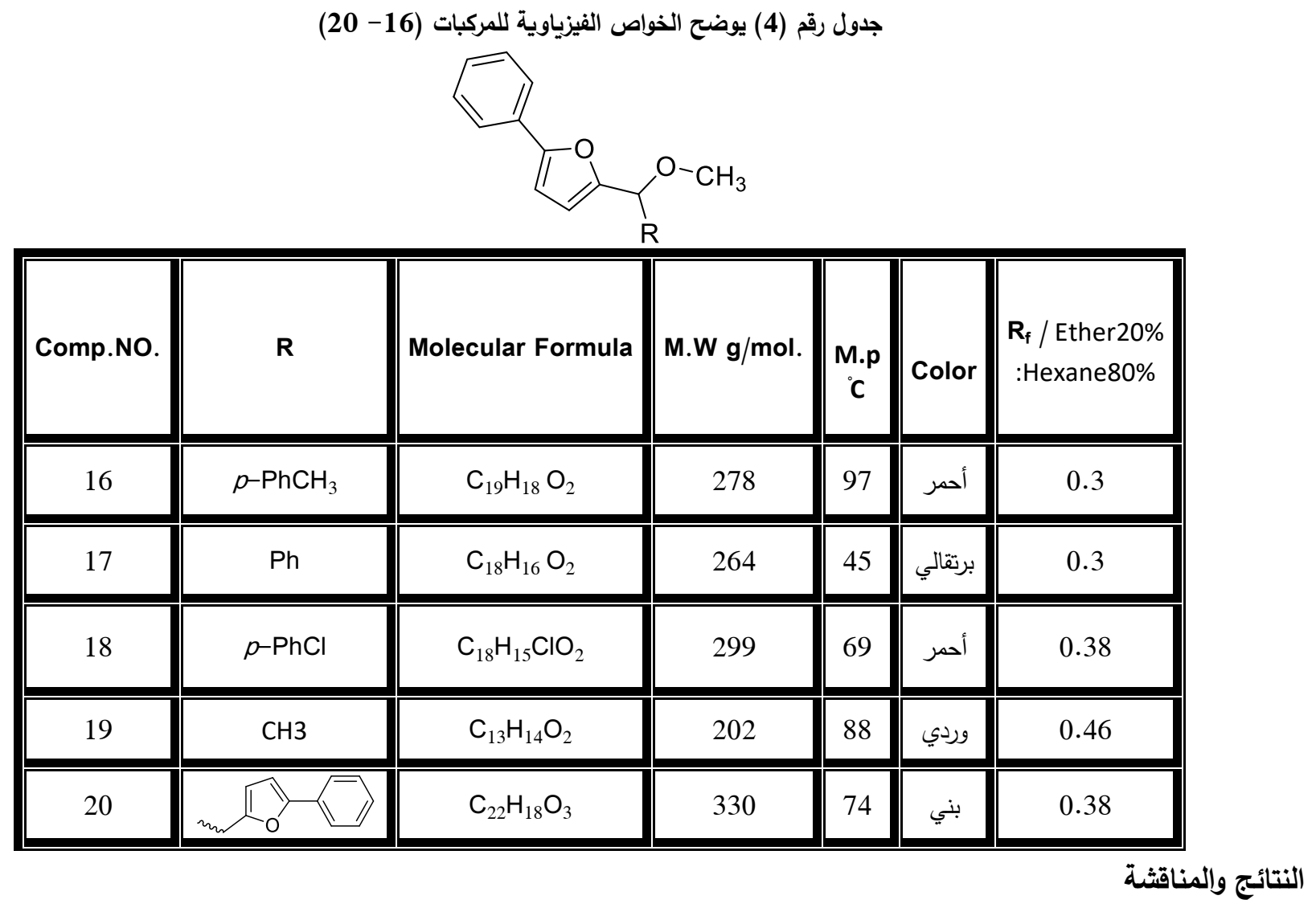

في هذا البحث استخدم السينامالديهايد كنواة لتحضير عدد من الاندوبيروكسيدات بواسطة تفاعل الاوكسجين الاحادي ومن

ثم تحويلها الى الفيورانات المقابلة كما في المخطط التالي (1)

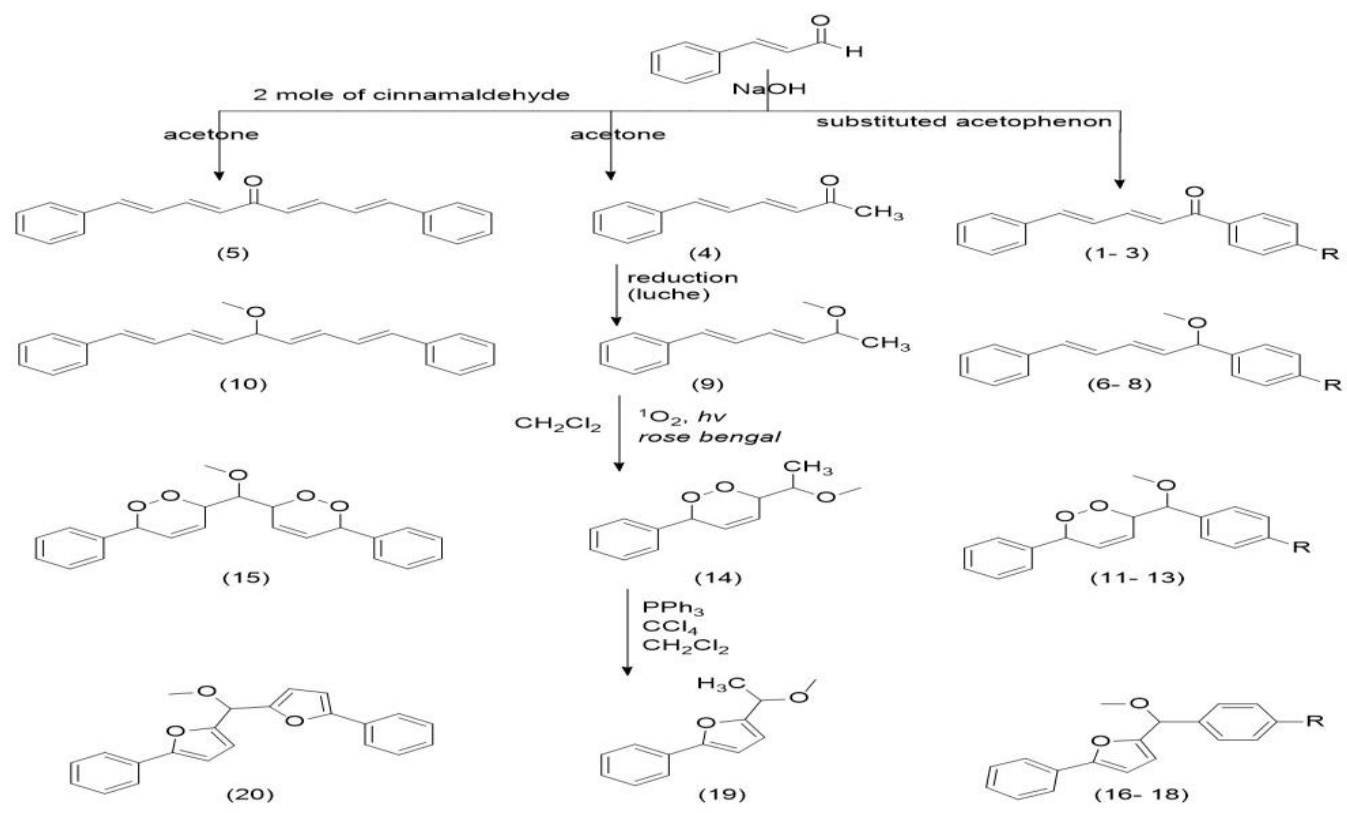

$\mathrm{R}=\mathrm{CH}_{3} \cdot \mathrm{H}, \mathrm{Cl}$

مخطط رقم (1) - (1) - (1) 
استخدم السينامالديهايد للتكاثف مع مول واحد من الكيتونات الاروماتية مثل الأسيتو فينون ومعوضاته وهكذا تم استخدام الاسيتون مرتين, الاولى استخدم مول من الاسيتون مع مول من السيناملديهايد, والثانية مول من الاسيتون مع مولين من السيناملديهايد لتكوين

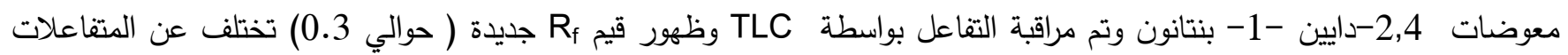
الى حين انتهاء التفاعل باختفاء المواد المتفاعلة كما في المعادلة التالية ومخطط رقم (1):<smiles>[R]C(=O)/C=C/C=C/c1cccc(C)c1</smiles>

ميزت المركبات (1- 3) بواسطة قياس درجة الأنصهار كما هو مبين في الجدول رقم (1) وشُخِصت المركبات بواسطة طيف الأشعة تحت الحمراء (IR) فقد أظهرت حزم مط تعود لمجموعة الكاربونيل بازاحة تتراوح بين 1650 الى 1677 ويعود سبب انخفاضها عن المعدل الطبيعي الى وجود مجموعتين من الاواصر المتعاقبة. والجدول أدناه يوضح الخواص الطيفية للمركبات المحضرة. بالنسبة للمركبات (4- 5) حضرت باستخدام الطريقة السابقة الذكر في وباستخدام الاسيتون مع مول واحد من السينلمالديهايد مره (4) ومرة اخرى مع مولين من السناملديهايد(5). شخصت المركبات المحضرة بالطرق الفيزياوية السابقة الذكر بالاضافة الى طيف الاشعة تحت الحمراءاذ اعطت ازاحات منخفضة لمجاميع الكاربونيل 1652 و 1649 سم -1 بسبب وجود الاواصر المتعاقبة كذلك حزم العائدة لاواصر المزدوجة حيث تراوحت بين 1571 الى 1602سم- الجدول التالي يوضح الخواص الطيفية [14 -113]. جدول(5) الخواص الطيفية (IR) (1-5).

\begin{tabular}{|c|c|c|c|c|c|c|}
\hline Comp.No. & $\mathrm{R}$ & $\begin{array}{l}\mathrm{C}-\mathrm{H} \\
\mathrm{Ar} .\end{array}$ & $\begin{array}{l}\text { C-H } \\
\text { Aliph. }\end{array}$ & $\mathrm{c}=\mathrm{O}$ & $\mathrm{C}=\mathrm{C}$ & other \\
\hline 1 & $p-\mathrm{PhCH}_{3}$ & 3024 & 2910 & 1650 & 1604,1575 & \\
\hline 2 & $\mathrm{Ph}$ & 3066 & - & 1652 & $\begin{array}{c}1597 \\
1579\end{array}$ & \\
\hline 3 & $p-\mathrm{PhCl}$ & 3055 & - & 1677 & $\begin{array}{l}1650 \\
1587\end{array}$ & $\begin{array}{l}\mathrm{C}-\mathrm{Cl} \\
1050\end{array}$ \\
\hline 4 & $-\mathrm{CH}_{3}$ & 3026 & 2925 & 1652 & $\begin{array}{c}1595 \\
1571\end{array}$ & \\
\hline 5 & & 3022 & - & 1649 & $\begin{array}{c}1602 \\
1568\end{array}$ & \\
\hline
\end{tabular}


يدعى التفاعل اعلاه بتفاعل تكاثف ألألدول وهو أحد أهم الطرق الاصطناعية التي تعطي جزيئات كبيرة من خلال تكوين روابط

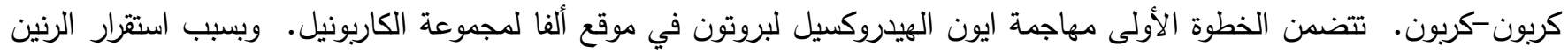
للقاعدة المترافقة (enolate) يعتبر هيدروجين حامضية عدد الفا هو الدفضل للهجوم النيكليوفيلي ، وفي الخطوة الثانية يهاجم الانايون

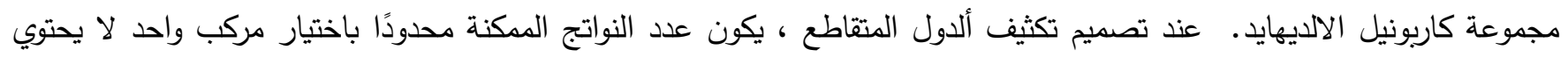

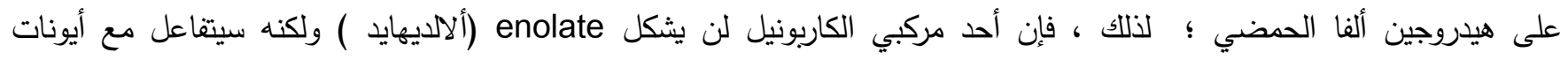

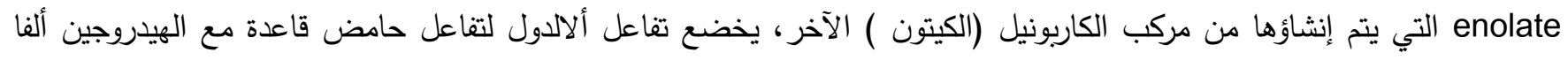

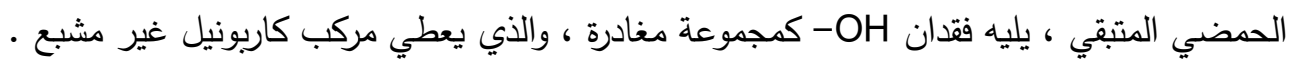

تم أختزال مجموعة الكاربونيل وتحويلها الى ميثوكسي بواسطة أختزالLuche وهو اختزال متخصص يستهدف مجموعة الكربونيل دان بيرها (الاصرة المزدوجة) للحصول على عدد من مركبات 4,2-دايين -1- ميثوكسي بنتان تم مراقبة التفاعل بواسطة وظهور قيم Rf جديدة تختلف عن المتفاعلات الى حين انتهاء التفاعل تم تثخيص المركبات بواسطة قياس درجة الأنصهار كما هو

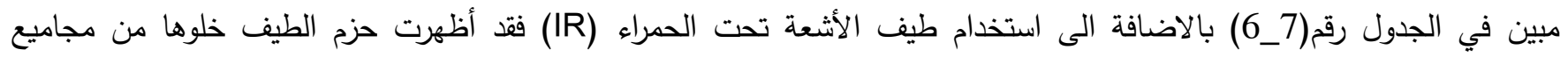
الكاربونيل وظهور مجاميع الميثوكسي اما المجاميع الاخرى فقد حصل لها تغيير طفيف في مقدار الإزاحة, الجدول أدناه يوضح الخواص الطيفية للمركبات المضرة<smiles>[R]C(=O)/C=C/C=C/c1ccccc1</smiles>

$(1-5)$

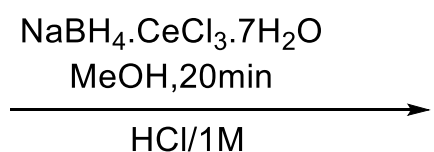

$\mathrm{HCl} / 1 \mathrm{M}$<smiles>[R]C(C=CC=Cc1ccccc1)OC</smiles>

$(6-10)$

جدول (6) الخواص الفيزيائية للمركبات (6- 10)

\begin{tabular}{|c|c|c|c|c|c|c|c|}
\hline Comp.No. & $\mathrm{R}$ & $\begin{array}{l}\mathrm{C}-\mathrm{H} \\
\text { Ar. }\end{array}$ & $\begin{array}{l}\text { C-H } \\
\text { Aliph. }\end{array}$ & $\mathrm{c}=\mathrm{C}$ & $\begin{array}{c}\mathrm{C}=\mathrm{C} \\
\text { aromatic }\end{array}$ & $\begin{array}{l}\text { C-O } \\
\text { Asy. }\end{array}$ & other \\
\hline 6 & $p-\mathrm{PhCH}_{3}$ & 3074 & 2931 & 1620 & 1480 & 1175 & \\
\hline 7 & $\mathrm{Ph}$ & 3137 & 2960 & 1660 & 1438 & 1105 & \\
\hline 8 & $p-\mathrm{PhCl}$ & 3052 & 2918 & 1640 & 1488 & 1127 & $\begin{array}{l}\mathrm{C}-\mathrm{Cl} / \\
793\end{array}$ \\
\hline 9 & $\mathrm{CH}_{3}$ & 3052 & 3024 & 1617 & 1491 & 1191 & \\
\hline 10 & & 3195 & 3010 & 1638, & 1456 & 1189 & \\
\hline
\end{tabular}

الميكانيكة المتترحة لتفاعل الاختزال (luche Reduction) باستخدام صوديوم رباعي هيدريد البورون مع العامل المساعد  مهمين في هذه الميكانيكية؛ الاول: هو تسهيل تكوين معقد الميثوكسي بروهايدرايد methoxybrohydride والثاني: هو زيادة الكتروفيلية 
ذرة كاربون مجموعة الكاربونيل عن طريق التناسق مع ذرة أوكسجين المذيب الذي يعمل على زيادة حامضية وسط التفاعل الذي بدوره يعمل على تنشيط كاربون الكاربونيل.

يعتمد نوع مجموعة الايثر على نوع الكحول المستخدم في التقاعل, شكل ( 2 ) يوضح تفاصيل هذه الميكانيكية [15]. $\mathrm{NaBH}_{4}+\mathrm{nMeOH} \longrightarrow \mathrm{CeCl}_{3} \longrightarrow \mathrm{NaBH}_{(4-n)}(\mathrm{OMe})_{n}+{ }_{n} \mathrm{H}_{2}$

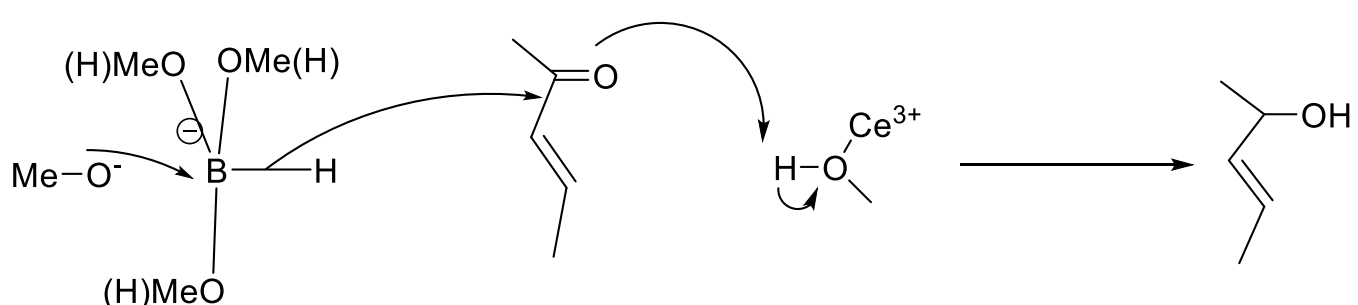

\section{شكل ( 2) ميكانيكية اختزال مجموعة الكاربونيل غير المشبعة}

تم تحويل مركبات (10-5-methoxypenta-1,3-diene(6 اللى الأندوبيروكسايد من خلال تفاعل الأوكسجين الأحادي بوجود صبغة الروز البنغالي rose Bengal(R.B وأستخدم ثنائي كلورو ميثان مرة و بعض المرات الكلوروفورم كمذيب بالاضافة الى لى تسليط سيل من فقاعات الاوكسجين خلال المذيب وبوجود الضوء المرئي, ان تفاعل الاوكسجين الاحادي Singlet Oxygen يعتبر من التفاعلات النظيفه كونه يحدث في الطبيعه بثكل واسع في كلوروفيل النبات وكذلك يتميز بقلة او انعدام النواتج الثانوية وسهولة تتقية النواتج وان التفاعل يحدث في ظروف متعادلة وبالإضافة الى امكانية استعمال الهواء الجوي كمصدر للاوكسجين [16]. تم مراقبة

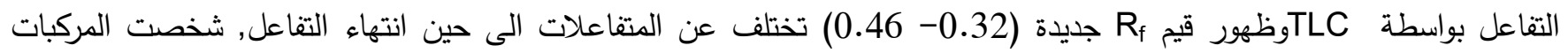
بواسطة قياس درجة الأنصهار حيث اعطت درجات تختلف عن مثيلاتها في الخطوة السابقة وكذلك بواسطة طيف الأشعة تحت الحمراء

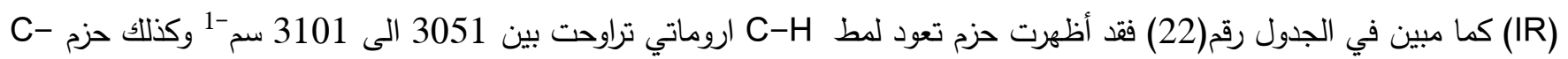
H الليفاتي تراوحت بين 2923 الى 3023 سم -1 وحزم C-O تراوحت بين

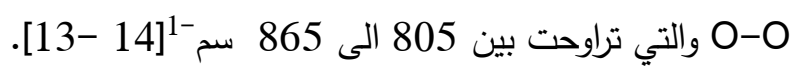
الجدول أدناه يوضح الخواص الطيفية للمركبات المحضرة<smiles>[R]C(/C=C/C=C/c1ccccc1)OC</smiles> 
جدول(7) الخواص الطيفية للمركبات (11- 15)

\begin{tabular}{|l||c||c||c|c|c||c||c||}
\hline $\begin{array}{l}\text { Comp. } \\
\text { No. }\end{array}$ & $\mathrm{R}$ & $\begin{array}{c}\mathrm{C}-\mathbf{H} \\
\text { Ar. }\end{array}$ & $\begin{array}{c}\mathbf{C}-\mathbf{H} \\
\text { Aliph. }\end{array}$ & $\mathbf{C}=\mathbf{C}$ & $\begin{array}{l}\mathbf{C}=\mathbf{C} \\
\text { aromatic }\end{array}$ & $\begin{array}{l}\text { C-O } \\
\text { Asy. }\end{array}$ & O-O \\
\hline \hline 11 & $p-\mathrm{PhCH}_{3}$ & 3053 & 2923 & 1623 & 1440 & 1112 & 811 \\
\hline \hline 12 & $\mathrm{Ph}$ & 3105 & 2925 & 1625 & 1438 & 1118 & 805 \\
\hline \hline 13 & $p-\mathrm{PhCl}$ & 3100 & 3029 & 1658 & 1465 & 1166 & 823 \\
\hline \hline 14 & $-\mathrm{CH}_{3}$ & 3101 & 2933 & 1660 & 1460 & 1184 & 808 \\
\hline \hline 15 & & 3159 & 2925 & 1625 & 1460 & 1103 & 865 \\
\hline
\end{tabular}

ان ميكانيكية تفاعل تكوين الاندوبيروكسايد تتبع الاضافة [4 +2] وهي تتبع ميكانيكية ديلزألدر كما في الثكل (4) حيث يكون التفاعل في حالة توازن مع المركب الوسطي إذ أضيفت جزيئة الأوكسجين من الجهة المقابلة لأواصر باي لتتكون حلقة سداسية كون الاضافة تكون لذرتي الاوكسجين على 3,1_diene (اربع ذرات كاربون) ليكون الناتج حلقة سداسية . هذه المركبات تمتلك خواص كيميائية جيدة كونها مركبات فعالة يمكنها التفاعل بسهولة لاعطاء عدد كبير من المركبات الطبيعية وغير الطبيعية المهمة بيولوجيا[17].

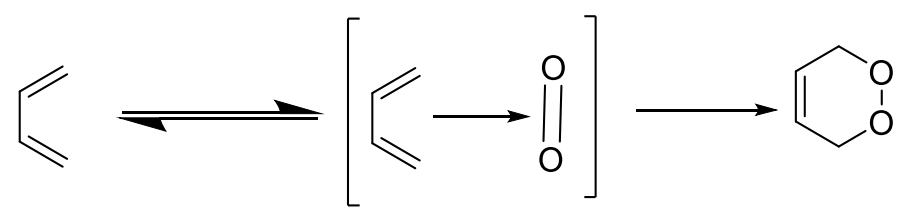

شكل (4) ميكانيكية الاضافة [4 +4 [2]

تم تحويل الاندوبيروكسيدات الىى الفيورانات المقابلة بتطبيق ظروف تفاعل ابل (Appel). تفاعل ابل يستخدم لتحويل الكحولات الى هاليدات الاكيل المقابلة, وفي عام 2017 تمكن الباحث M. Kimber نجح في تطبيق ظروف التفاعل على الاندوبيروكسيدات لتعاني من فقدان جزيئة ماء لتحول الى الفيوران[5] ـ شخصت المركبات المحضرة بواسطة ال TLC لاثبات ان التفاعل اعطى ناتج جديد يختلف عن المواد المتفاعلة وظهور قيم Rf جديدة (0.3- 0.46) بالاضافة الى ان النواتج تمتلك درجات انصهار تختلف عن المركبات السابقة. كما شخصت النواتج بواسطة طيف ال IR حيث اعطت حزم متوسطه تعود لمط C-H اروماتي تتراوح بين (3150 (20) الى 3028 ) سمَّ حزم $29-H$ C-H اليفاتي تراوح بين 2916 الى 3062 سم - بالاضافة الى حزم هيكلية تعود لحلقتي الفيوران والبنزين كما

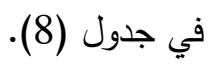
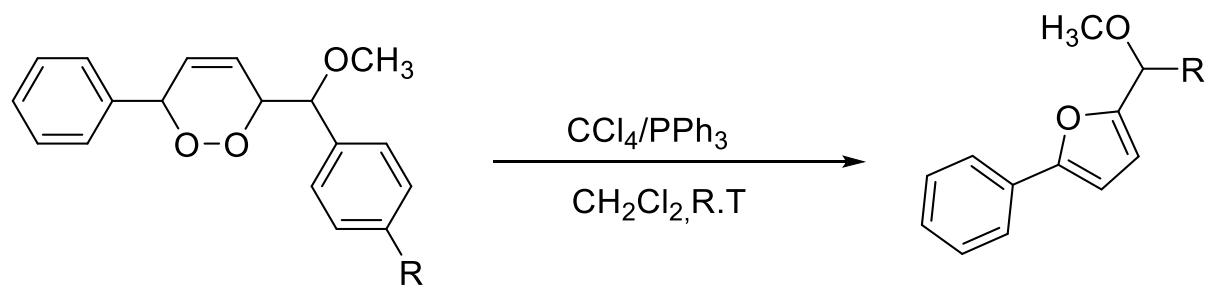
جدول رقم (8) الخواص الطيفية للمركبات (16 - 20)

\begin{tabular}{||c||c||c|c|c||l|l||}
\hline Comp.No. & $\mathrm{R}$ & $\begin{array}{c}\text { C-H } \\
\text { Ar. }\end{array}$ & $\begin{array}{c}\text { C-H } \\
\text { Aliph. }\end{array}$ & $\mathbf{C}=\mathbf{C}$ & C-O & other \\
\hline \hline 16 & $p-\mathrm{PhCH}_{3}$ & 3028 & 2958 & 1676,1514 & 1118 & \\
\hline \hline 17 & $\mathrm{Ph}$ & 3105 & 3062 & 1625,1595 & 1188 & \\
\hline \hline 18 & $p-\mathrm{PhCl}$ & 3087 & 2985 & 1630,1593 & 1182 & $\mathrm{C}-\mathrm{Cl} / 723$ \\
\hline \hline 19 & $-\mathrm{CH}_{3}$ & 3101 & 2950 & 1627,1598 & 1164 & \\
\hline \hline 20 & -1 & 3150 & 2927 & 1625,1598 & 1118 & \\
\hline
\end{tabular}

كما شخصت النواتج بواسطة طيف ال HNNMR للمركبات ألنهائية وقد اظهرت ألنتائج ان جميع المركبات اعطت حزمتين ثنائيه كل منهما تعود الى احد بروتوني حلقة الفيوران عند المدى

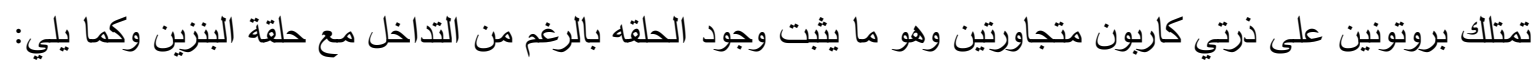
Compound 16 : ${ }^{1} \mathrm{H}$ NMR (ppm DMSO-d6) $\delta$ 7.6- 7.55(m, 9H), 7.26(d ,1H), 6.99(d , 1H), 6.09(s, 1H), $3.35(\mathrm{~s}, 3 \mathrm{H}), 2.32(\mathrm{~s}, 3 \mathrm{H})$. Compound 17: ${ }^{1} \mathrm{H}$ NMR (ppm DMSO-d6) $\delta$ 7.66- 7.55 (m, 10H), 7.15(d,1H), 7.00(d, 1H), 6.09( S, 1H), $3.43(\mathrm{~s}, 3 \mathrm{H})$.

Compound 18: ${ }^{1} \mathrm{H}$ NMR (ppm DMSO-d6) $\delta$ 7.66- 7.55 (m, 9H), 7.13 (d, 1H), 6.98(d, 1H), 6.09(s, 1H), $3.43(\mathrm{~s}, 3 \mathrm{H})$.

Compound 19: (ppm; DMSO-d6) $\delta$ 7.64- $7.42(\mathrm{~m}, 5 \mathrm{H}), 7.12(\mathrm{~d}, 1 \mathrm{H}), 6,63(\mathrm{~d}, 1 \mathrm{H}), 4.92(\mathrm{~s}, 1 \mathrm{H}), 1.57(\mathrm{~s}$, 3H).

Compound 20: ${ }^{1} \mathrm{H}$ NMR (ppm; DMSO d6) $\delta$ 7.82- $7.40(\mathrm{~m}, 10 \mathrm{H}), 7.43(\mathrm{~d}, 1 \mathrm{H}), 7.35(\mathrm{~d}, 1 \mathrm{H}), 7.25(\mathrm{~d}$ ,1H), $6.63(\mathrm{~d}, 1 \mathrm{H}), 6.15(\mathrm{~s}, 1 \mathrm{H}), 3.51(\mathrm{~s}, 3 \mathrm{H})$.

تضمنت الميكانيكية المقترحة من قبل الباحث المترحة ان يهاجم المزدوج الالكتروني في ثلاثي فنيل فوسفين ذرة كلور في رباعي

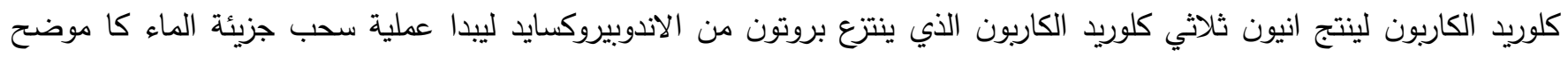
المخطط (5) ادناه [5]. 

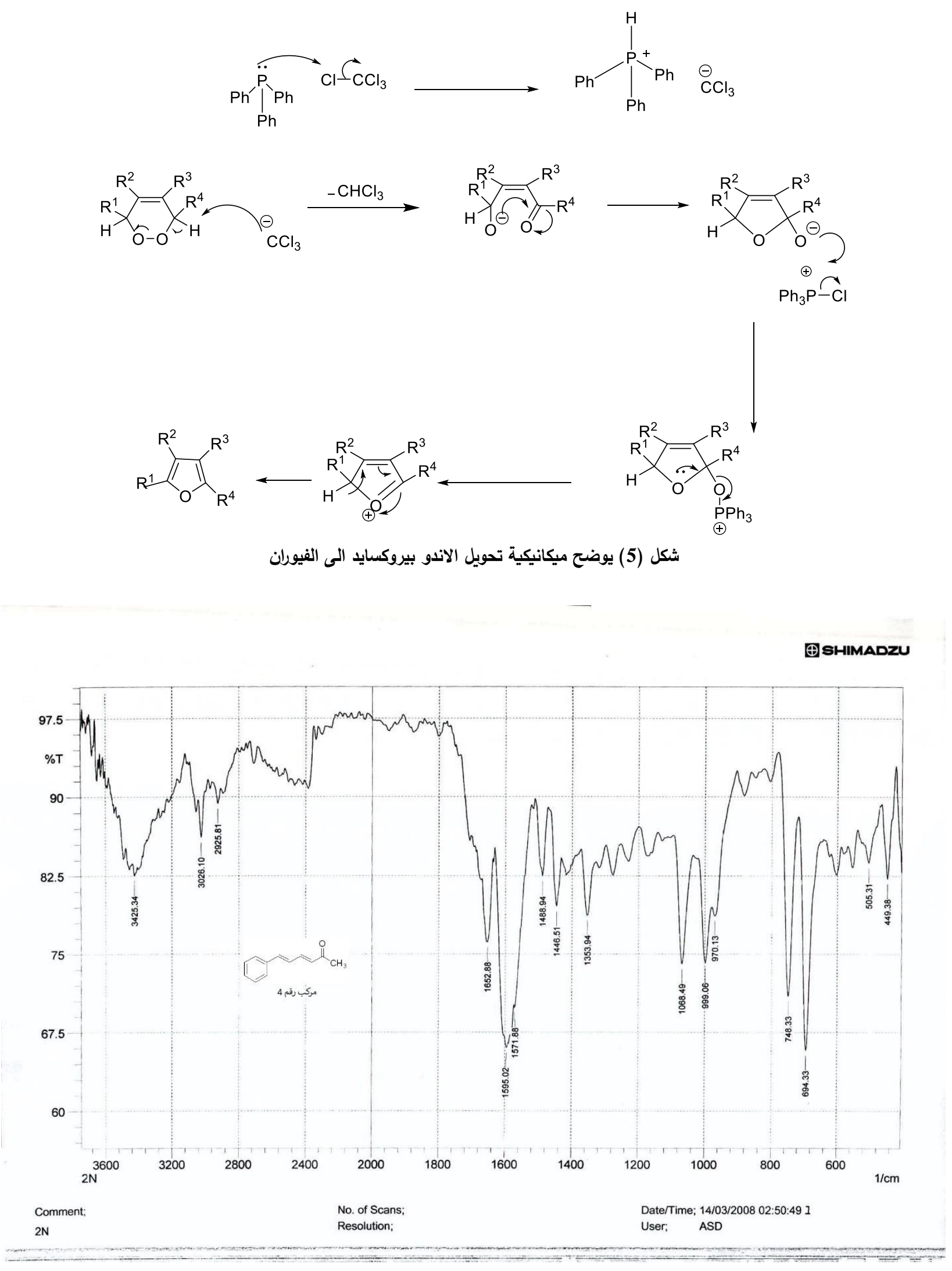

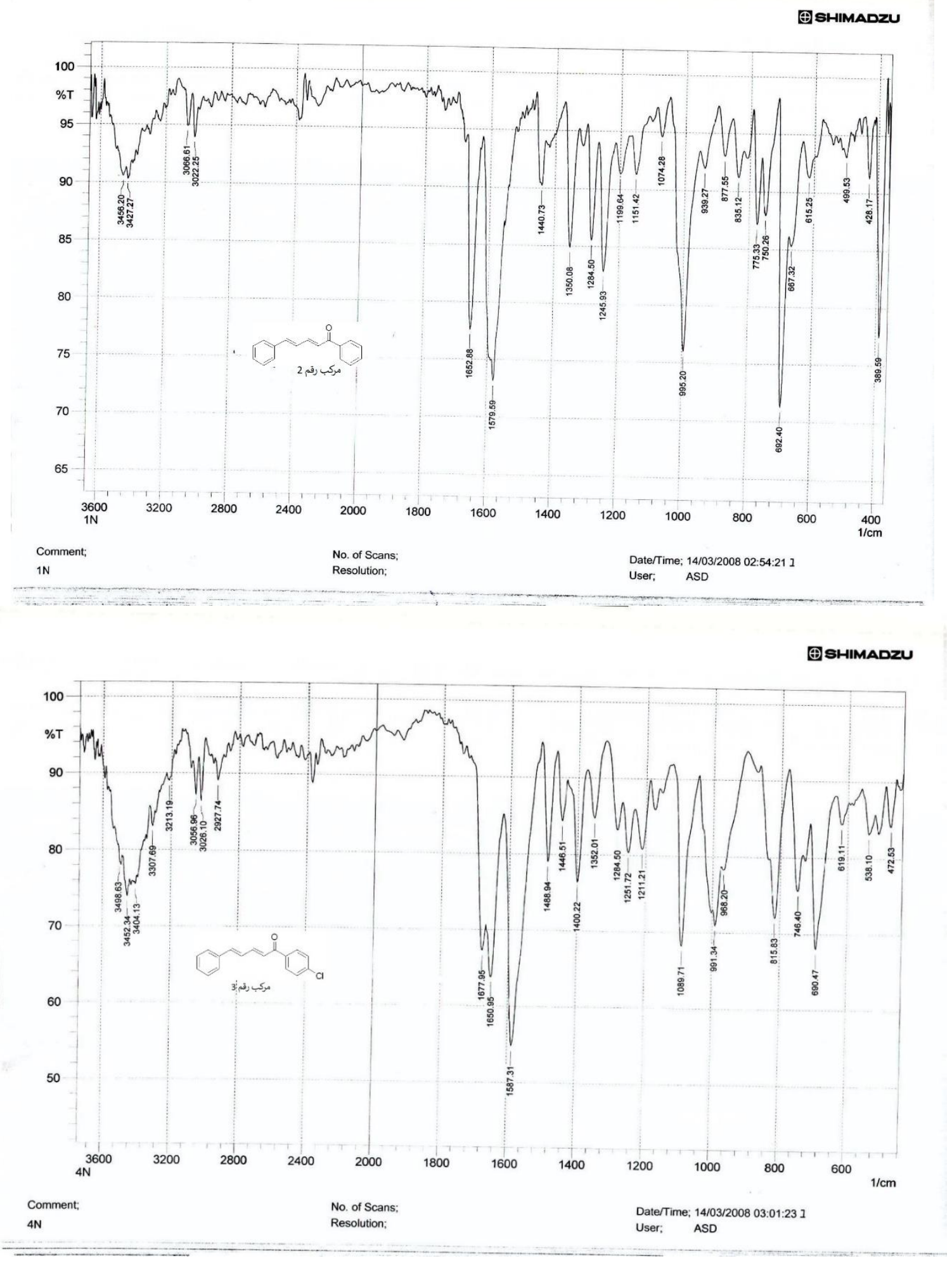
Journal of Education and Science (ISSN 1812-125X), Vol: 30, No: 5, 2021 (147-162)

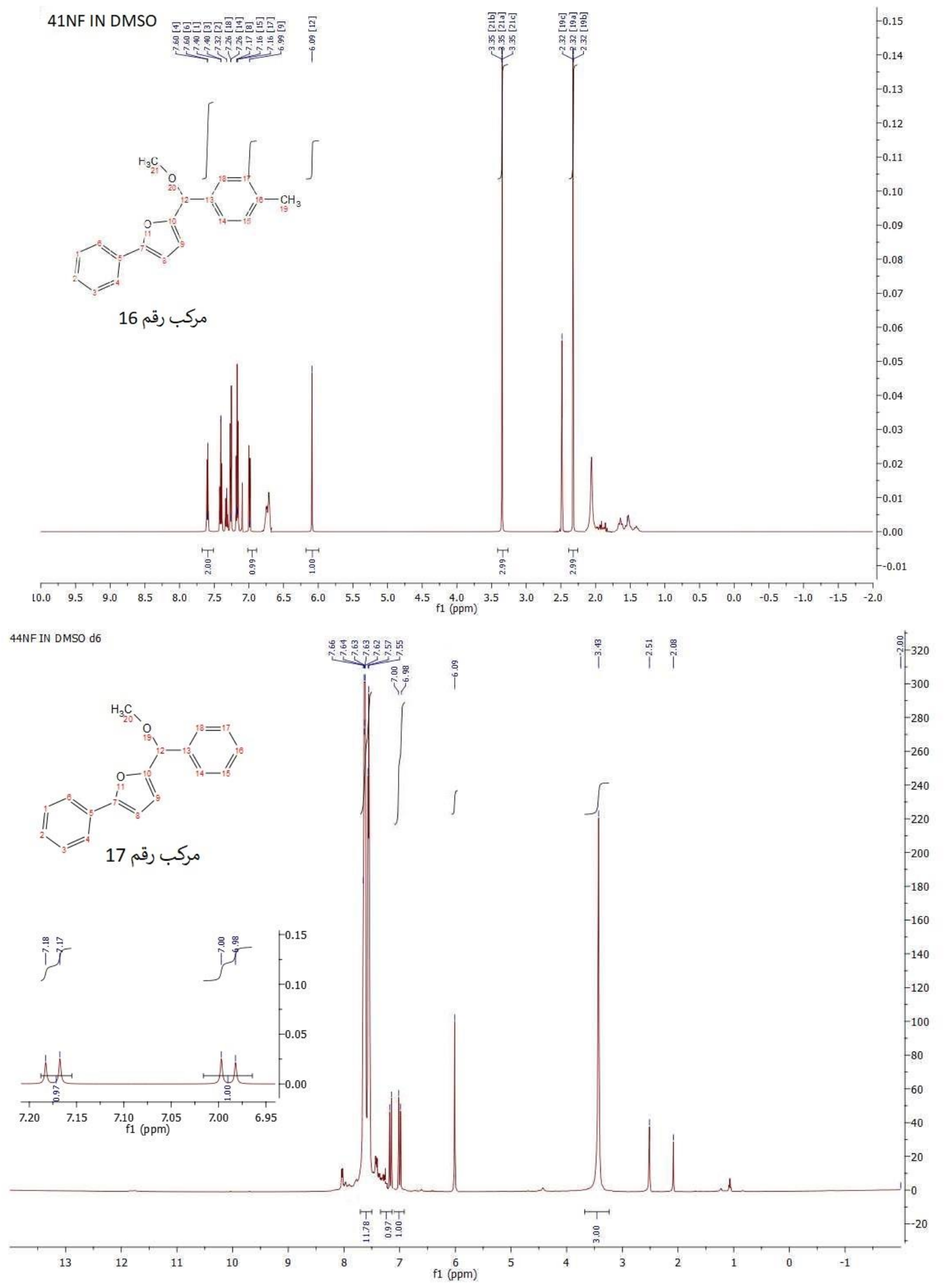




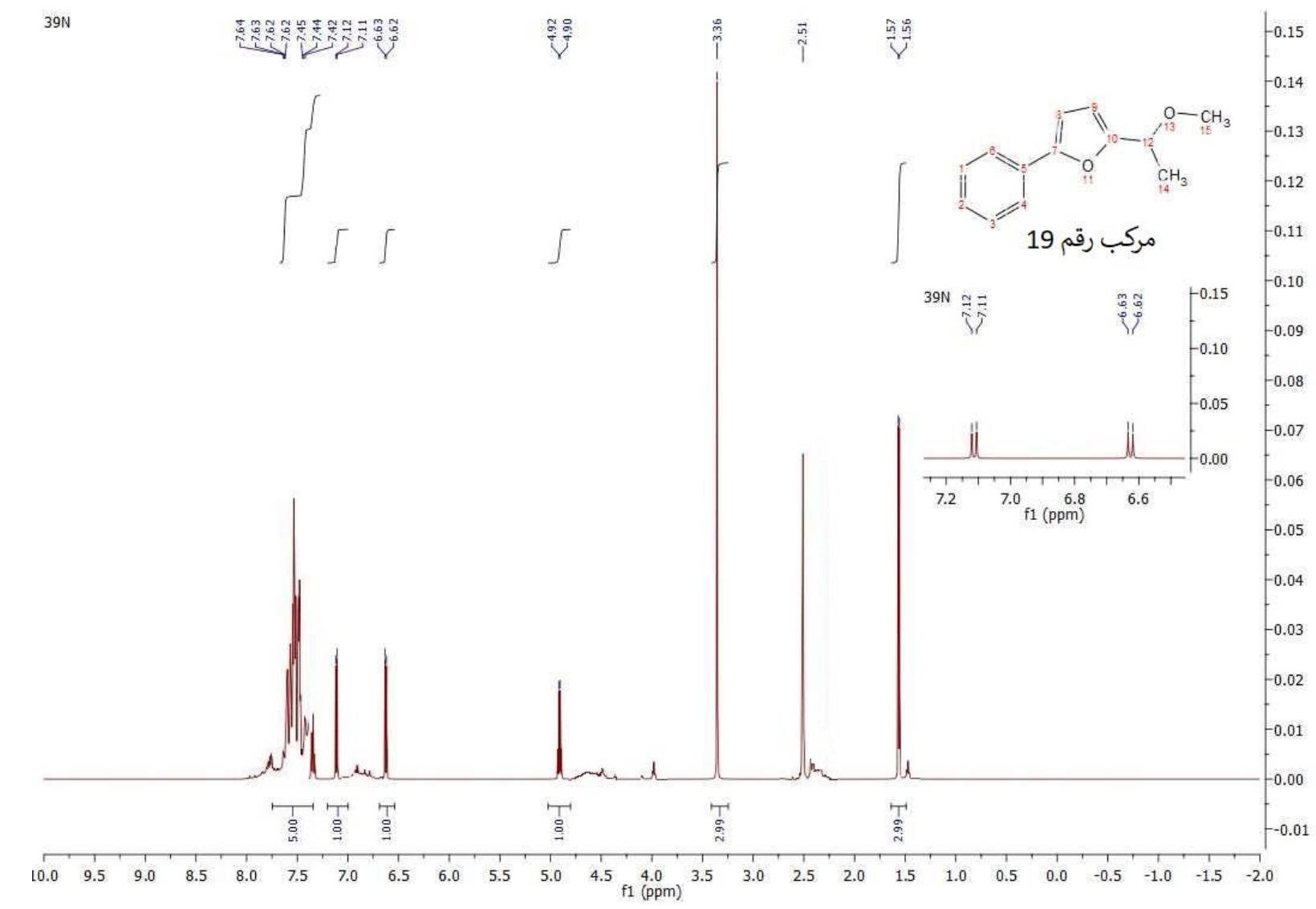

الثكر والتقدير

نتقدم بالثكر والتقدير الى كلية التربية للعلوم الصرفة /جامعة الموصل لاتاحتها لنا فرصة للعمل في مختبرات بحوث الكيمياء العضوية

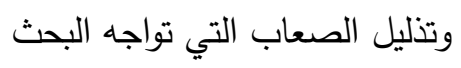

المصادر :

1- G. Lipan; Martindale - the Extra Pharmacopoeia' (30th ed), edited by J. E. F. Reynolds, 1993, p 221-226.

2- Lopz F, Jett M, Mchowski JM, Nitzn D and Yang C, Synthesis, and biological evaluation of Keterolc analogs. Heterocycles, 2002, 56, 91-97.

3- Meotti FC, Silv DO, Sntos ARS, Zeni G, Rocha JBT and Nogueira CW, Thiophenes and furans derivatives: a new class of potential pharmacological agents. Environmental Toxicology and Pharmacology, 2003, 37, 37-44.

4- Wsley J. Moran and Arntxa Rodríguez; Metal-catalyzed Furan Synthesis. A Review; Organic Preparations and Procedures International, 2012, Volume 44, Issue 2, 103-130.

5- Robert J. Lee,a Martn R. Lndley,b Gareth J. Pritcharda and Marc C. Kimber*; A biosynthetically inspired route to substituted furans using the Appel reaction: total synthesis of the furan fatty acid F5; r, Chem. Commun., 2017, DOI: 10.1039/C7CC03229C.

6- Dong, Z.; Liu, J.; Wang, F. and Liu, J. Two Novel Norssquiterpene Peroxides from Basidiomycete Stccherinum Ochaceum. Tetrahedron Lett. 2010, 51, 3152-3153.

7- Rudrpal, M.; Cheta, D. Endoperoxide Antimalarials: Development, Structural Diversity and Pharmacodynamic Aspects with Reference to 1,2,4-Trioxane-Based Structural Scaffold. Drug Des. Devel. Ther. 2016, 10, 3575-3590. 
8- Ghogare, A. A., \& Greer, A. Using singlet oxygen to synthesize natural products and drugs. Chemical Reviews, (2016), 116(17), 9994-10034.

9- Gao-Fei Pan, Xng-Long Zhang, Xue-Qing Zhu, Rui-Li Guo, and Yong-Qiang Wang; Synthesis of (E,E)-Dienones and (E,E)-Dienals via Palladium-Catalyzed g,d-Dehydrogenation of Enones and Enals; iScience 20, 2019, 229-236.

10- Nicoletta Desderi, Rossella Fioravanti, Luca Proietti Monaco, Mariangela Biava, Matilde Yáñez, Francesco Ortuso, Stefano Alcro; 1,5-Diphenylpenta-2,4-dien-1-ones as potent and selective monoamine oxidase-B inhibitors; European Journal of Medicinal Chemistry, 2013, 59, 91-100.

11- Philpp Fruhmann, Christian Hametner, Hannes Mikula, Gerhard Adam, Rudolf Krska and Johannes Fröhlich; Stereoselective Luche Reduction of Deoxynivalenol and Three of Its Acetylated Derivatives at C8; Toxins 2014, 6, ISSN 2072-6651.

12- Salamci, E. A Concise and Stereospcific Synthesis of Some Cyclitols Containing Eight Membered Rings: Cyclooctane-1,2,3,4-Tetraoles. Tetrahedron 2010, 66 (23), 4010-4015.

13- Robert M. Silverstin, Francis X. Webster and David J. Kiemle; Spectrometric Identification Of Organic Compounds; State University of New York, 2005, p 91, John Wiley 8« Sons, Inc.

14- Erno Prtsch, Philippe Buhlmann and Martin Badertscher; Structure Determination of Organic Compounds, Fourth, Revised and Enlarged Edition, 2009, p 291, Springer-Verlag Berlin Heidelberg.

15- Gemal, A. L., Luche, J. L. Lanthanoids in organic synthesis. Reduction of $\alpha$-enones by sodium borohydride in the presence of lanthanoid chlorides: synthetic and mechanistic aspects. J. Am. Chem. Soc. 1981, 103, 5454-5459.

16- Alxis J. Eugene and Marcelo I. Guzman; Production of Singlet Oxygen $\left({ }^{1} \mathrm{O}_{2}\right)$ during the Photochemistry of Aqueous Pyruvic Acid: The Effects of $\mathrm{pH}$ and Photon Flux under Steady-State $\mathrm{O}_{2}$ (aq) Concentration; Environ. Sci. Technol. 2019, 53, 21, 12425-12432.

17- Eske, A.; Goldfus, B.; Griesbek, A. G.; De Kiff, A.; Kleczka, M.; Leven, M.; Neudörfl, J. M.; Vollmer, M. Ene-Diene Transmissive Cycloaddition Reactions with Singlet Oxygen: The Vinylogous Gem Effect and Its Use for Polyoxyfunctionalization of Dienes. J. Org. Chem. 2014, 79 (4), 1818 1829. 